Research Article

\title{
Three-Dimensional Structure Analysis of Urban Landscape Based on Big Data Technology and Digital Technology
}

\author{
Xiaozhou Yang ${ }^{1}$ and Fan Bai $\mathbb{D}^{2}$ \\ ${ }^{1}$ Department of Art, Northeastern University, Shenyang 110006, China \\ ${ }^{2}$ School of Equipment Engineering, Shenyang Ligong University, Shenyang 110159, China \\ Correspondence should be addressed to Fan Bai; snowwind001@sylu.edu.cn
}

Received 26 September 2021; Revised 11 October 2021; Accepted 30 October 2021; Published 12 November 2021

Academic Editor: Hai Dong

Copyright ( $) 2021$ Xiaozhou Yang and Fan Bai. This is an open access article distributed under the Creative Commons Attribution License, which permits unrestricted use, distribution, and reproduction in any medium, provided the original work is properly cited.

\begin{abstract}
In order to improve the effect of urban landscape design, this paper combines big data technology with digital technology. For scenes and solutions containing SDS paths, a processing method similar to photon graphs is used and added to the calculation of two-way optical path tracking. In the processing scene, this paper uses the two-way optical path tracking method to perform specular reflection or refraction from the subpath starting from the light source and then store information such as the light energy of the points on the diffuse reflection surface or the directional reflection surface. Moreover, this paper combines the actual needs of urban landscape design to construct an urban landscape design system based on big data technology and digital technology. Finally, this paper designs experiments to carry out urban landscape simulation and design effect evaluation. From the test results, it can be seen that the system designed in this paper basically meets the needs of urban landscape planning and design.
\end{abstract}

\section{Introduction}

Place spirit is the core concept of place theory, and the two spiritual attributes of direction and identity together constitute place spirit. Direction refers to people's ability to clarify the relationship between themselves and the environment, and identity refers to people's identification with themselves through meaningful connections with the surrounding environment in the place, which is a spiritual affirmation and belonging [1]. In modern society, people are unfamiliar and alienated from their living environment and lose their sense of identity. That is why they promote the generation of lost space. During the design process, attention is paid to the analysis of the needs of the crowd, and after the investigation and analysis, the spatial transformation to cater to the living environment preferred by the residents around the site would inevitably make the lost space regain its vitality. The use of a positive place structure and a rich place spirit can create a place where people are happy and unwilling to move. It will inevitably promote the establishment of place spirit, and the problem of lost space spirit loss can be solved [2].
In the process of transforming and designing the lost space of urban landscape, the creation of spatial ambiguity is mainly to create diversified activity spaces and rationally lay out the five elements of urban space: roads, boundaries, regions, nodes, and landmarks. From the overall perspective, it meets the psychological and behavioral needs of different groups of people and provides facilities for people to stop, talk, and rest. The facilities and space design can meet the requirements of different activities. Moreover, the design should analyze the social crowd activities of all age groups, so that the space can serve all kinds of people. In the space design, various spaces are planned in a unified manner, and a diverse landscape space is created to meet the conversion of functions. In the creation of details, the design can provide public facilities with multiple functions, landscape sketches for people to play and participate, vertical tree ponds, etc. [3].

Due to the severe lack of interaction and communication with users, lost spaces often give people a negative impression of darkness and chaos, and they are not attractive to people. In the redesign of the lost space, it attracts people to 
have a full range of perceptual experience of the space environment, mobilizes the enthusiasm and interest of people to participate in activities, attracts people to integrate into the environment, and promotes a good interactive communication between people and the landscape, emphasizing space. The continuity and fluidity of the "lost" can be dispelled, and the beautiful enjoyment brought by the space environment can be felt from all angles. Once people develop a habit of this feeling, it can effectively extend the user's stay in the landscape space. Through the transformation of leisure and entertainment methods, the interactive landscape has enhanced public communication and adjusted according to public feedback to make its own functional form more perfect. For example, the fountain facilities and interesting landscape sketches in the square enable people to directly touch and appreciate them at close range, thus leaving a good memory for people's travel, which can easily promote people's reexperience and effectively add space to the space. Vitality. Another design method is to use hightech materials and technology to create a changing and dynamic space effect. New things are more likely to attract people's attention, so as to attract people's extensive participation, mobilize people's enthusiasm, and activate the atmosphere of the place, thereby enhancing the landscape, and the vitality of the space.

This paper combines big data technology and digital technology to analyze the three-dimensional structure and performance of urban landscapes and provides a theoretical reference for subsequent urban landscape design.

\section{Related Work}

The $3 \mathrm{D}$ reconstruction technology based on multiview geometry [4] needs to use the projection relationship between the image sequence obtained from different perspectives in the same scene and the target scene to restore the geometric spatial position of each image feature in the image sequence, so as to achieve the three-dimensional reconstruction of the geometric model of the target scene. Generally, three-dimensional reconstruction is achieved based on multiple cameras simultaneously shooting scene sequence images from different perspectives, or based on monocular cameras time-sharing shooting scene image sequences from different perspectives. Multiview geometric 3D reconstruction mainly solves some problems in the motion recovery structure method, such as feature matching and viewpoint correspondence [5]. The multiview geometric method usually includes four main steps: image feature extraction and matching, calculation of multiview geometric constraint relations, iterative optimization of estimation results, and dense reconstruction of sparse scenes. Literature [6] proposed that when the spatial relationship between two projections is unknown, the related perspective projection relationship is described to realize a simple algorithm for reconstructing the three-dimensional structure of a scene. The corresponding problem of image points in different perspectives in the same scene is solved. When the image matching problem is solved, the $3 \mathrm{D}$ reconstruction of the scene can be simplified to find the relative direction of the two viewpoints, that is, the geometric constraint relationship. Since then, the three-dimensional reconstruction based on the multiview motion restoration structure kicked off. Literature [7] conducted a detailed study on the nature of the essential matrix that can express the geometric constraint relationship. Literature [8] proposed the basic matrix, explained its basic properties, and solved the geometric constraint relationship between two images. The concept of the basic matrix was promoted, and the theory of multiview geometry continued to develop and grow. Literature [9] proposed the Scale Invariant Feature Transform (SIFT) algorithm. The local features extracted by the algorithm remain unchanged against rotation, scale scaling, and brightness changes, and they also maintain a certain degree of stability against viewing angle changes, affine transformations, and noise. The emergence of this algorithm makes the problem of matching between images from different perspectives in the same scene better solved. The first two steps of the multiview geometric reconstruction process have been solved, and the initial reconstruction results have been obtained at this time. In order to optimize the initial three-dimensional reconstruction results, modern methods often use the bundle adjustment method (BA) to optimize the cost function of the total reprojection error. The cost function can be used to determine the model parameters and camera pose of each camera at the same time, so that the difference between the image measurement value and the model prediction value can be minimized. This step is the most critical in large-scale $3 \mathrm{D}$ reconstruction [10].

Literature [11] uses the sparse beam adjustment method as the underlying optimization engine to incrementally reconstruct the target three-dimensional scene by inputting the disordered image set, image feature points, and their matching results. With the development of multicore processors and GPUs, the GPU-based SIFT algorithm SiftGPU [12] appeared. In addition, an efficient beam adjustment method based on the conjugate gradient method has been proposed one after another, called Multicore Bundle Adjustment (MBA) [13]; on the basis of both MBA and SiftGPU, Wu once again proposed an incremental SFM software package called VisualSFM [14]. VisualSFM not only has a good graphical interactive interface, but also visualizes each step of the SFM, allowing users to clearly understand its operation process. The most important thing is to obtain the output camera's motion posture, trajectory, and reconstruction from the input image sequence. The three-dimensional scene structure completely realizes the process steps of SFM from beginning to end [15]. The estimation of the 3D model in SFM is usually calculated by calculating the spatial position of the feature points from the static scene image to generate a point cloud with the target model structure as the output result, but the structure of the point cloud is sparse, and it is impossible to complete some of the real society. Requirements, such as $3 \mathrm{D}$ printing, so the sparse model needs to be densified [16]. Based on multiview stereo vision (multiview stereopsis (MVS)) theory, literature [17] proposed patch-based multiview stereo software (PMVS), which combines sparse key points into a block structure and performs three steps of matching, expansion, and filtering. 
Processing gets more matching points and generates dense point cloud. When the data set is large, the amount of calculation in the intensive reconstruction process is huge, which causes the operation to be time-consuming. In order to improve the computing efficiency, the new version PMVS2 is optimized on the basis of PMVS, which has improved speed and quality [18]. Clustering views for multiview stereo (CMVS) [19] includes PMVS2 and some additional features. It not only groups and clusters the input image data set, but also processes multiple data sets in parallel to achieve faster and dense reconstruction under large data sets. It also removes redundant image data and improves the accuracy of reconstruction. Literature [20] believes that although the traditional SFM method based on point feature detection and matching can obtain the correct camera pose, the number of available matching features is small, and the number of accurate 3D space points is extremely limited. Although MVS solves this problem, however, the computational complexity is relatively high. In view of the rich linear features of the building, a reconstruction method based on the straight line segment as the bottom layer feature is proposed, and pure geometric constraints are used to match the straight line segments in different images to achieve the reconstruction of the building, and geometric structure reconstruction.

\section{Big Data and Digital Landscape 3D Structure Design Algorithm}

The ray tracing method is a collection of methods that can actually display objects. The ray casting algorithm is the prototype of the ray tracing method. The algorithm idea is to track the light that reaches the viewpoint in the opposite direction and go through any pixel of the screen to find the first object that intersects the opposite light. After that, it calculates whether the object is illuminated by the light source and finally returns the color value of the intersection point. The method of ray projection only considers the direct illumination of the object, so the reflection, refraction, penumbra, and other effects of the light on the surface of the object are difficult to present. Methods such as ray tracing improve the ray casting method.

Flux: the total energy of light passing through a certain surface or area in unit time. The unit is watts (W) and is usually represented by $w$.

Irradiance: the density of light energy passing through a surface or area in a unit of time. Its unit is $\left(\mathrm{W} / \mathrm{m}^{2}\right)$ and is usually represented by $E$, namely,

$$
E=\frac{\mathrm{d} \Phi}{\mathrm{d} A} \text {. }
$$

Intensity: the light energy within a unit solid angle in a given direction in a unit time. Its unit is $\left(\mathrm{W} / \mathrm{m}^{2}\right)$ and is usually represented by $I$, namely,

$$
I=\frac{\mathrm{d} \Phi}{\mathrm{d} \omega} .
$$

Radiance: the light energy within a unit solid angle in a given direction passing through a vertical unit area in unit time. Its unit is $\left(\mathrm{W} /\left(\mathrm{m}^{2} \cdot \mathrm{sr}\right)\right)$ and is usually expressed by $L$, namely,

$$
L=\frac{\mathrm{d} \Phi}{\mathrm{d} \omega \cdot \mathrm{d} A^{\perp}} .
$$

Among them, $L_{0}\left(p, \omega_{0}\right)=L_{e}\left(p, \omega_{0}\right)+\int$ is the projection of $\mathrm{dA}$ in the $\omega$ direction.

The rendering equation was first proposed by James Kajiya. It is an approximate expression of Maxwell's electromagnetic formula, and it removes the processing of diffraction phenomenon that is basically irrelevant to the display effect in Maxwell's equation.

The rendering equation is expressed as

$$
L_{0}\left(p, \omega_{0}\right)=L_{e}\left(p, \omega_{0}\right)+\int_{\delta^{2}}\left(p, \omega_{0}, \omega_{i}\right) L_{i}\left(p, \omega_{i}\right)\left|\cos \theta_{i}\right| \mathrm{d} \omega_{i} .
$$

Among them, $p$ is a point on the surface of the object, $\omega_{0}$ is the direction of the emitted light on the unit sphere, and $\omega_{i}$ is the direction of the incident light. $L_{i}\left(p, \omega_{i}\right)\left|\cos \theta_{i}\right|$ is the influence of the self-luminescence on the surface of the object on the emitted light. The integral form of $\int_{\delta^{2}}\left(p, \omega_{0}, \omega_{i}\right) L_{i}\left(p, \omega_{i}\right)\left|\cos \theta_{i}\right| \mathrm{d} \omega_{i}$ is calculated as the influence of incident light in all directions of the hemispherical surface $\left(\delta^{2}\right)$ on the outgoing light. Among them, $\left(p, \omega_{0}, \omega_{i}\right)$ is the two-way reflection distribution function.

Figure 1 uses a two-dimensional picture to illustrate the meaning of the rendering equation. The surface of the object seen at the viewpoint position is transmitted through the $\omega_{0}$ direction. In order to calculate the amount of light energy from this direction, it is necessary to know all the light energy at the location of the $\mathrm{P}$ point, and then according to the total light energy of the $\mathrm{P}$ point and the $\omega_{0}$ direction, the light energy received by the viewpoint can be obtained. The light energy at the $\mathrm{P}$ point may come from two situations:

(1) Self-luminous objects

(2) Other light-emitting surfaces or reflective (refracting) surfaces transfer light energy to the $\mathrm{P}$ point

The above two cases are solved separately and added together to get the total light energy at the point $\mathrm{P}$.

The physical meaning of the bidirectional reflectance distribution function (BRDF) refers to the ratio of the radiation micro-increment to the radiation micro-increment in the reflection direction. When the material of the object, the angle of reflection, and the angle of incidence are determined, BRDF is calculated as a constant. It is defined as follows:

$$
\begin{aligned}
\left(p, \omega_{0}, \omega_{i}\right) & =\frac{\mathrm{d} L_{r}\left(p, \omega_{0}\right)}{\mathrm{d} E_{i}\left(p, \omega_{i}\right)} \\
& =\frac{\mathrm{d} L_{r}\left(p, \omega_{0}\right)}{L_{i}\left(p, \omega_{i}\right) \cos \theta_{i} \mathrm{~d} \omega_{i}} .
\end{aligned}
$$




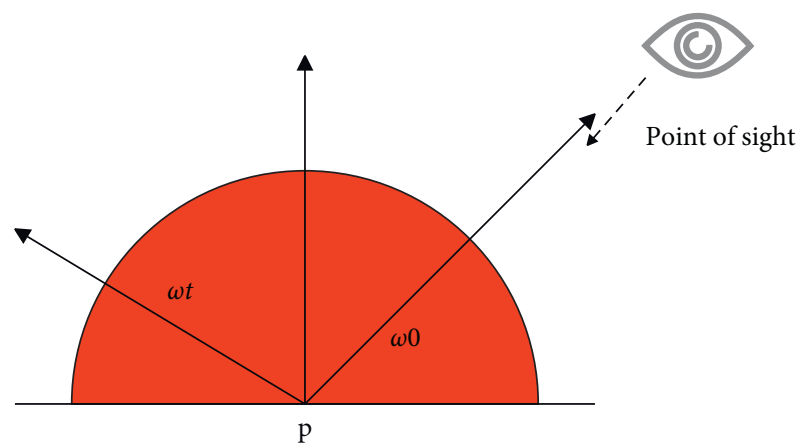

FIGURE 1: Basic reflection/refraction model.

Among them, $L$ is the luminous flux per unit steradian and $E$ is the luminous flux per unit area. $\theta_{i}$ is the angle between $\omega_{i}$ and the normal vector, as shown in Figure 2.

BRDF is expressed as the relationship between incident and reflection of an object. However, this is not enough to describe the propagation process of light. Among them, a more common situation is that after the light intersects the object and has an effect, part of it will be decomposed into reflected light, but the other part will be further transmitted into the object, as shown in Figure 3.

This part of the light energy may be absorbed by objects (Fresnel Conductors, such as some metals) and may further propagate downward (such as glass) and exist as refracted light. Therefore, when dealing with the light propagating inside the object, it is necessary to define the bidirectional transmittance distribution function (BTDF) and use it for processing.

According to different objects, the performance of reflected (refraction) light varies. We can divide the reflection (refraction) models of objects into the following categories.

3.1. Mirror Reflection/Refraction. The reflection (refraction) is concentrated in one direction. For mirrors, the reflection angle is equal to the incident angle, as shown in Figure 4.

3.2. Diffuse Reflection/Refraction. It is also called Lambert reflection. When the object is sufficiently rough, the brightness of the reflected light is constant in the $2 \pi$-space of the target point. That is, the reflected brightness does not change with the change of the observer's line of sight, as shown in Figure 5.

3.3. Directional Reflection/Refraction. It is also called nonLambert reflection. This reflection is between diffuse reflection and specular reflection. In this model, the brightness of the reflected light in each direction is different, as shown in Figure 6.

It is available that, for the above several types of reflection and refraction models, in addition to specular reflection, the direction of the reflection angle can be clearly known according to the angle of incidence. The other two types have countless possibilities in the direction of the reflection or refraction angle under the condition of a clear angle of incidence. Conversely, considering the situation of reverse ray tracing, under the condition of a certain viewing angle, according to the rendering equation, there are countless directions of light energy that affect the point and then affect the final color of the point.

Since the solution is an integral equation, and the integral parameters are uncertain, this equation cannot be calculated in one time. It can only be approximated by sampling. Under the condition of increasing number of samples, the approximate result is getting closer and closer to the correct solution.

The definition of Monte Carlo method is as follows.

The integral equation $\int_{a}^{b} f(x) \mathrm{d} x$ is calculated by sampling method, and the integral value is calculated by Monte Carlo method as

$$
F_{N}=\frac{b-a}{N} \sum_{i=1}^{N} f\left(X_{i}\right) .
$$

In the above equation, $N$ points are sampled in the integration range, and the solution of the integral equation is estimated with the sampling value of these $N$ points.

Now, we prove that if there is enough $N, F_{N}$ is approximately equal to $\int_{a}^{b} f(x) \mathrm{d} x$; that is, prove $E\left[F_{N}\right]=\int_{a}^{b} f(x) \mathrm{d} x$.

$$
\begin{aligned}
E\left[F_{N}\right] & =E\left[\frac{b-a}{N} \sum_{i=1}^{N} f\left(X_{i}\right)\right] \\
& =\frac{b-a}{N}\left[\sum_{i=1}^{N} E\left[f\left(X_{i}\right)\right]\right] \\
& =\frac{b-a}{N}\left[\sum_{i=1}^{N} \int_{a}^{b} f(x) \frac{1}{b-a} \mathrm{~d} x\right] \\
& =\frac{1}{N}\left[\sum_{i=1}^{N} \int_{a}^{b} f(x) \mathrm{d} x\right] \\
& =\int_{a}^{b} f(x) \mathrm{d} x .
\end{aligned}
$$

When $f(x)$ cannot be expressed by an explicit equation (such as solving a rendering equation), the Monte Carlo method is a good choice. The method mentioned above is to sample randomly within the integration interval. However, if we can roughly know the curve shape of $f(x)$, we can formulate a probability distribution function $p(x)$ based on the approximate curve trend of $f(x)$. Moreover, we set the curve shape that is more suitable for $f(x)$ and perform sampling and calculation according to the following formula:

$$
F_{N}=\frac{1}{N} \sum_{i=1}^{N} \frac{f\left(X_{i}\right)}{p\left(X_{i}\right)}
$$

In the case of sufficient $N, F_{N}$ is approximately equal to $\int_{a}^{b} f(x) \mathrm{d} x$. However, the sampling strategy is based on $p(x)$, and there are more samples in the peak part of $p(x)$. If $p(x)$ fits the shape of $f(x)$ very well, then a smaller number of 


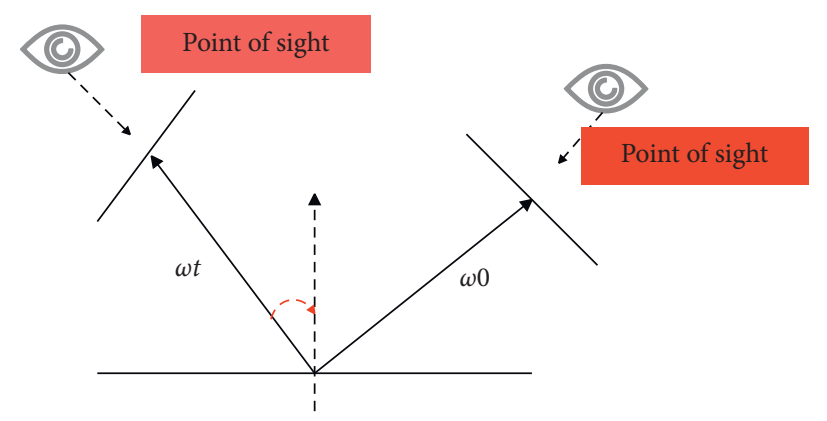

FIGURE 2: Distribution function of bidirectional reflection.

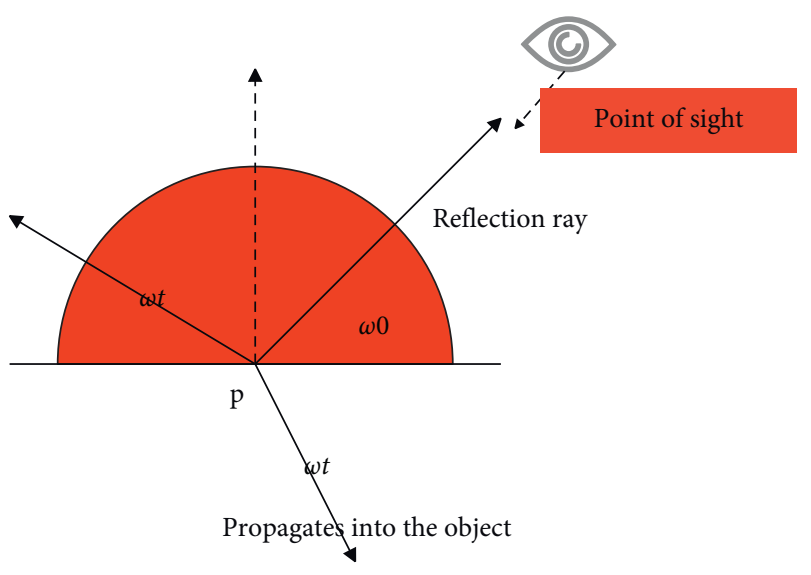

FIGURE 3: The propagation mode of light.

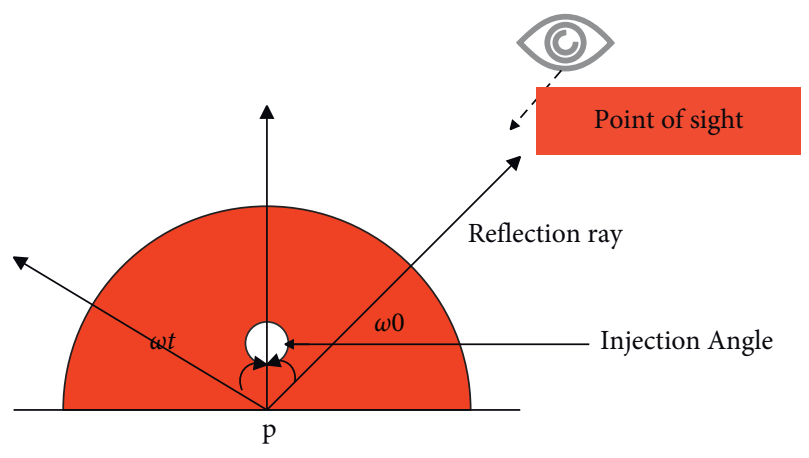

FIgURE 4: Specular reflection model.

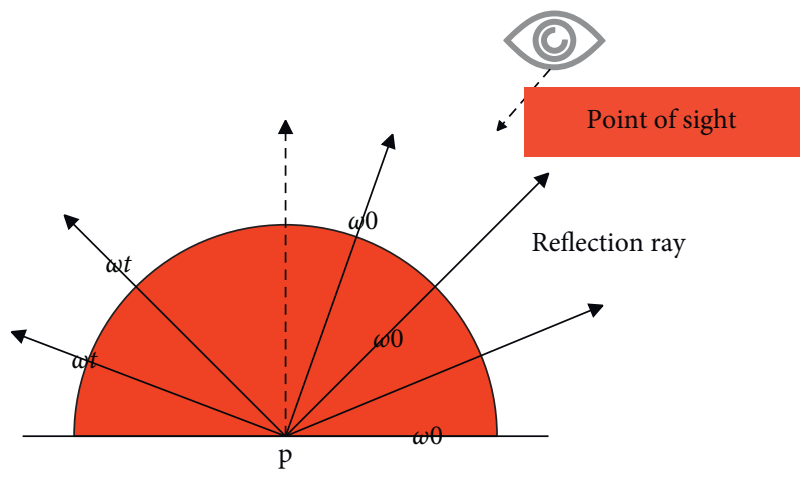

FIGURE 5: Diffuse reflection model.

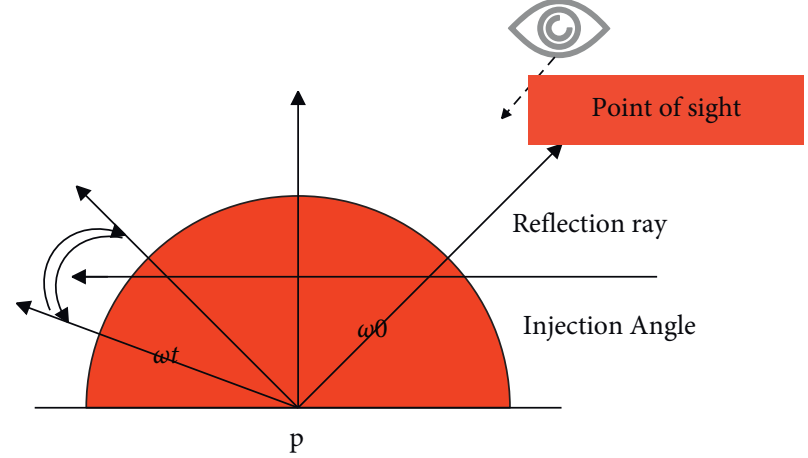

FIgURE 6: Directional reflection model.

samples can be used to get a more similar result to $\int_{a}^{b} f(x) \mathrm{d} x$.

According to the general Monte Carlo method, we can apply it to the solution of rendering equations. This is also the basis of all the following solution algorithms. For example, for diffuse reflection model or directional reflection model,

$$
\begin{aligned}
L_{0}\left(p, \omega_{0}\right) & =L_{e}\left(p, \omega_{0}\right)+\int_{\delta^{2}}\left(p, \omega_{0}, \omega_{i}\right) L_{i}\left(p, \omega_{i}\right)\left|\cos \theta_{i}\right| \mathrm{d} \omega_{i} \\
& =L_{e}\left(p, \omega_{0}\right)+\frac{1}{N} \sum_{i=1}^{N} \frac{\left(p, \omega_{0}, \omega_{i}\right) L_{i}\left(p, \omega_{i}\right)\left|\cos \theta_{i}\right|}{p\left(\omega_{i}\right)} .
\end{aligned}
$$

For non-SDS paths, the integral solution method is the same as the bidirectional optical path tracking. The specific method is described below.

Figure 7 is a schematic diagram of two-way optical path tracking. In the schematic diagram, there are two sublight paths, $P 1^{\prime} \longrightarrow P 2^{\prime} \longrightarrow P 3^{\prime}$ from the line of sight, and $P 1^{\prime}$ $>P 2^{\prime}->P 3^{\prime}$ from the light source. It can be seen from the figure that these suboptical paths can form up to $3 * 3$, and a total of 9 complete optical paths are available for use. Now, we connect $P 3$ and $P 2^{\prime}$ and assume that there is no obstruction between these two points, and the planes where the two points are located are both diffuse reflection surfaces. At this time, the light starts from the light source and passes through $P 1^{\prime}, P 2^{\prime}, P 3, P 2$, and $P 1$ and finally reaches the line of sight position. This constitutes a complete optical path, so that this complete path will affect the light energy at point $P 1$ 


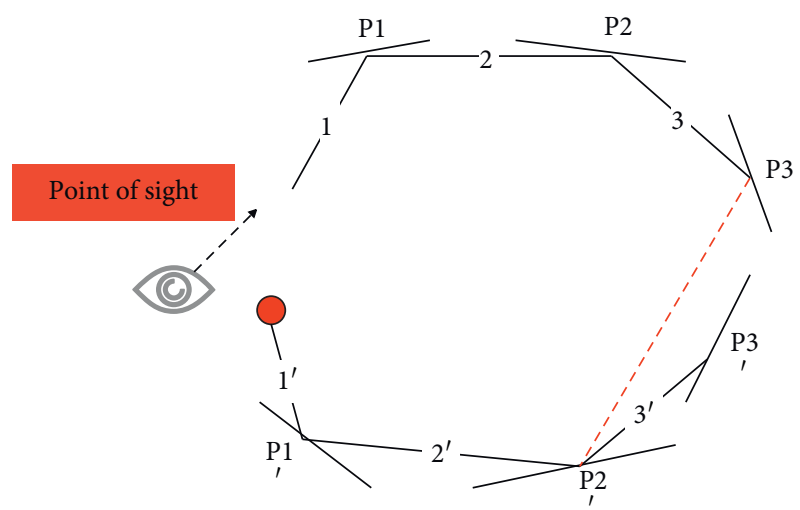

FIgURE 7: Schematic diagram of two-way optical line tracking.

and eventually return to the line of sight position via line 1 . The formula is expressed as follows: In this way, we can get the specific color value received by the line of sight.

$$
\begin{aligned}
C= & \frac{\operatorname{Le}\left(P_{0}^{\prime}, P_{0}^{\prime} \longrightarrow P_{\prime_{1}}\right)}{p\left(P_{0}^{\prime}, P_{0}^{\prime} \longrightarrow P_{1}^{\prime}\right)} \cdot\left(\frac{P_{1}^{\prime}, P_{1}^{\prime} \longrightarrow P_{0}^{\prime}, P_{1}^{\prime} \longrightarrow P_{1}^{\prime}, P_{2}^{\prime}}{p\left(P_{0}^{\prime} \longrightarrow P_{2}\right)}\right) \cdot\left(P_{2}^{\prime}, P_{2}^{\prime} \longrightarrow P_{1}^{\prime}, P_{2}^{\prime} \longrightarrow P_{3}^{\prime}\right) . \\
& \left(P_{3}, P_{3} \longrightarrow P_{2}^{\prime}, P_{1} \longrightarrow P_{2}\right) \cdot\left(\frac{\left(P_{2}, P_{2} \longrightarrow P_{3}^{\prime}, P_{2} \longrightarrow P_{1}\right)}{p\left(P_{2} \longrightarrow P_{3}\right)} \cdot \frac{\left(P_{1}, P_{1} \longrightarrow P_{2}, P_{2} \longrightarrow P_{0}\right)}{p\left(P_{1} \longrightarrow P_{2}\right)}\right) .
\end{aligned}
$$

First, we know the two sublight paths $P \mathrm{I} \longrightarrow P 2 \longrightarrow P 3$ and $P 1^{\prime} \longrightarrow P 2^{\prime} \longrightarrow P 3^{\prime}$ and define the light source sampling position as Po' and the line of sight sampling position as P0. According to the Monte Carlo method, according to the probability distribution function at the light source, the light energy value of the auspicious point is divided by the probability distribution function value here. At position $P_{2}^{\prime}$, according to the probability distribution function at $P_{2}^{\prime}$, the BRDF is also divided by the value of the probability distribution in the sampling direction $P_{1}^{\prime} \longrightarrow P_{2}^{\prime}$. Then, the light energy throughput from position $P_{2}^{\prime}$ to position $P_{3}$ is calculated by BRDF, and the calculation is performed up to the viewpoint position. The result $\mathrm{C}$ is the final light energy received by the viewpoint.

When calculating the energy of the light path, the weight of the light path needs to be considered. During the light path tracking process, the same light path may be repeated many times. For example, at a certain moment, the following situation may occur.

In Figure 8, the generated path is the same as that in in Figure 7 . The only difference is that they are obtained from different subpaths.

Therefore, for each complete path, a certain weight needs to be added. We assume that there is a complete path 1 , and its length is $n$. As long as the suboptical path from the line of sight (assuming the length is $s$ ) and the suboptical path from the light source (assuming the length as $t$ ) satisfy $s+t-1=n$, path 1 may be generated. What needs to be ensured is that, in all cases, $s$ and $t$ satisfy the sum of their weights to 1 .

Eric Veach explained, on the basis of experiments, that the best weighting equation is as follows:

$$
\begin{aligned}
w(s, t) & =\frac{p_{j}^{\beta}}{\sum_{i=0}^{s+t-1} p_{i}^{\beta}} \\
& =\frac{1}{\sum_{i=0}^{s+t-1}\left(p_{i}^{\beta} / p_{j}^{\beta}\right)} .
\end{aligned}
$$

Among them, $p_{j}$ refers to the probability distribution function. On this basis, Eric Veach pointed out that the equation performs best when the index is $\beta=2$.

In the actual calculation process of the two-line optical path tracking, if calculated according to the original weighting equation, it will consume a lot of time. After modifying the original equation, a simple calculation method can be obtained. If it is assumed that pi is expressed as the length of the subpath starting from the line of sight in the total optical path with a total length of $n$, then 


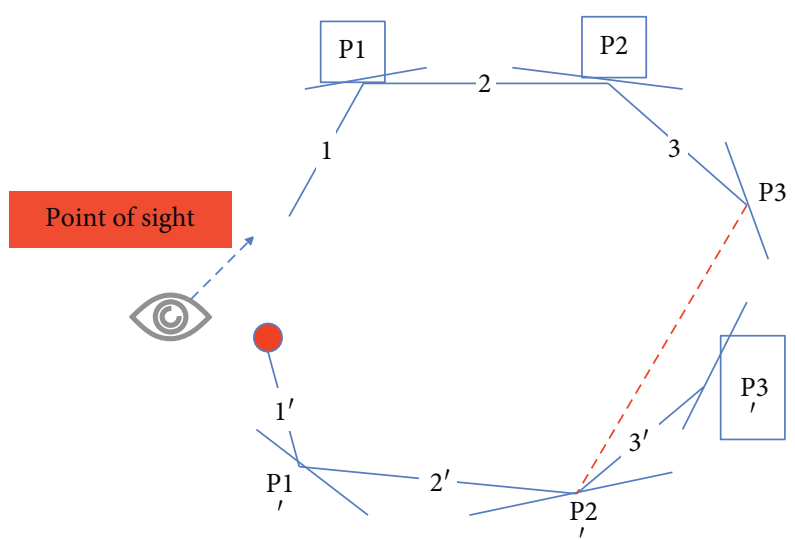

FIgURE 8: Schematic diagram of the weight problem.

$$
\begin{aligned}
\frac{p_{i+1}}{p_{i}} & =\frac{p\left(e_{0} \longrightarrow e_{1}\right) \cdot p\left(e_{1} \longrightarrow e_{2}\right) \cdots p\left(e_{i-1} \longrightarrow e_{i}\right)}{p\left(e_{0} \longrightarrow e_{1}\right) \cdot p\left(e_{1} \longrightarrow e_{2}\right) \cdots p\left(e_{i-2} \longrightarrow e_{i-1}\right)} \\
& =\frac{p\left(l_{0} \longrightarrow l_{1}\right) \cdot p\left(l_{1} \longrightarrow l_{2}\right) \cdots p\left(l_{n-i-2} \longrightarrow l_{n-i-1}\right)}{p\left(l_{0} \longrightarrow l_{1}\right) \cdot p\left(l_{1} \longrightarrow l_{2}\right) \cdots p\left(l_{n-i-1} \longrightarrow l_{n-i}\right)} \\
& =\frac{p\left(e_{i-1} \longrightarrow e_{i}\right)}{p\left(l_{n-i-1} \longrightarrow l_{n-i}\right)} .
\end{aligned}
$$

Thus, we can get the result of any $p_{i} / p_{j}$ from the result of $p_{i+1} / p_{i}$. For example,

$$
\frac{p_{i+2}}{p_{i}}=\frac{p_{i+2}}{p_{i+1}} \cdot \frac{p_{i+1}}{p_{i}}
$$

The simplicity of this method lies in the fact that the vertex results of the subpath in the middle of a bidirectional optical path tracing all need to be reused. In this way, the ratio of $p_{i+1} / p_{i}$ can be stored in the vertex structure, and only a few multiplications are required each time the weight is calculated.

For the SDS path, the two-way optical path tracking will completely ignore this type of path. At this time, it is necessary to use a method similar to the photon graph to process the SDS path for calculation. Such a method will bring a certain deviation to the result. In terms of robustness, this hybrid method is not as good as any unbiased method. However, this method can reuse the subpath starting from the light source, and the use of the vertices on these subpaths is not a pure two-way optical path tracking to calculate whether there is a speed block between any two points. Each calculation must traverse the entire ray acceleration structure, and its traversal speed is related to the scene complexity. The processing method similar to the photon graph is only to search for the vertex position related to the current calculation process, so the calculation efficiency of this method will be relatively high.

The common photon map method to calculate the light energy of the current point ( $N$-nearest) is as follows:

$$
L\left(P, \overrightarrow{\omega_{0}}\right) \approx \sum_{j=1}^{n} \frac{\left(P, \overrightarrow{\omega_{I J}}, \overrightarrow{\omega_{0}}\right) \phi_{j}\left(P_{j}, \overrightarrow{\omega_{I J}}\right)}{\pi r^{2}} .
$$

For the light energy of $\mathrm{P}$ position with direction $\overrightarrow{\omega_{0}}$, it is to multiply the light energy of all known points in the circle with radius $r$ by BRDF and statistically estimate the light energy of $\mathrm{P}$ position with direction $\overrightarrow{\omega_{0}}$, as shown in Figure 9.

The gray point is the required $\mathrm{P}$ position. It will look for all photons within the radius $r$ around itself and estimate the light energy at its own location. It is easy to get that the smaller the $r$, and the more the photons in the range of $r$, the closer the light energy of the final position of $\mathrm{P}$ to the light energy that $\mathrm{P}$ correctly carries. For the final image produced, the larger $r$ and the smaller the number of photons, the more blurred the image. On the contrary, the image is clearer and closer to the correct result. Of course, the solution time is longer.

Modifying the calculation method of the photon diagram, the solution method of the SDS path can be obtained. We assume that there is an SDS path scenario as shown in Figure 10.

At this time, it is necessary to add all the points within the radius of $r$ to the process of calculating the light energy of the diffuse reflection point. In the above figure, the viewpoint is traced down through the mirror position $P_{1}$, and light is emitted to a point $P_{1 e}$ on the diffuse reflection surface. The light source traces down through the mirror position $P_{2}$ and emits light to point $P_{2 l}$ on the same diffuse reflection surface. When we need to calculate the brightness of point $P_{1 e}$, we need to consider $P_{2 l}$. When point $P_{2 l}$ is within the range of $P_{1 e}$ with radius $r$, point $P_{2 l}$ is included in the calculation range.

$$
\begin{aligned}
& L\left(P_{1 e}, \omega_{0}\right) \approx\left(P_{1 e}, P_{1 e} \longrightarrow P_{1}, P_{1 e} \longrightarrow P_{2}\right) \\
& \cdot \phi\left(P_{2 l}, P_{2 l} \longrightarrow P_{2}\right) \cdot \frac{1}{\pi r^{2}} .
\end{aligned}
$$

As with the photon graph method, these $P_{2 l}$-type points can be reused. 


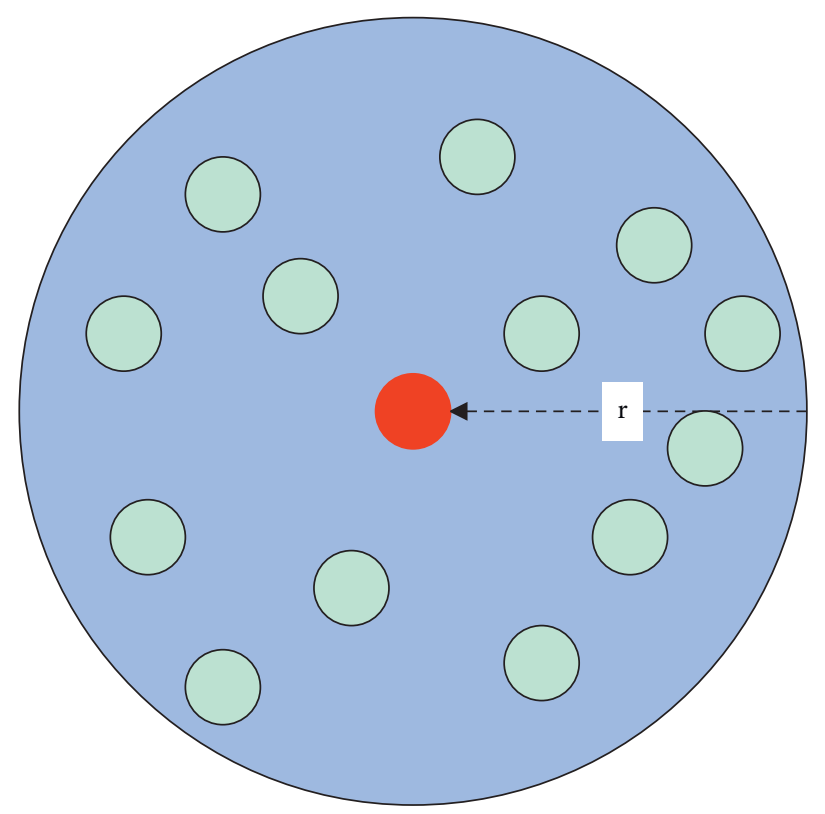

Figure 9: The way the photon map method calculates the rendering equation.

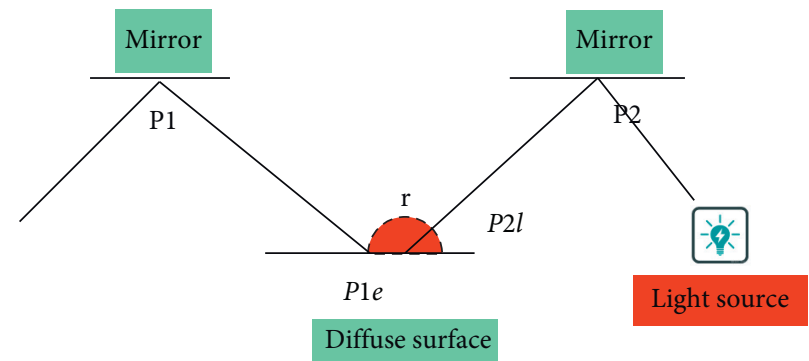

FIGURE 10: Dealing with SDS issues.

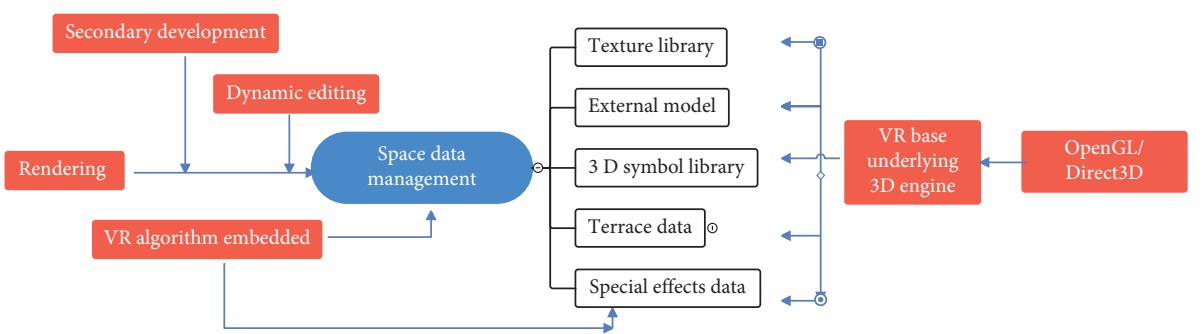

FIgURE 11: System structure diagram of the urban three-dimensional landscape basic platform system.

\section{Three-Dimensional Structure Analysis of Urban Landscape Based on Big Data Technology and Digital Technology}

To build a three-dimensional landscape platform, we need to understand its system composition. The architecture can reflect the operation flow and data flow. The system structure of the 3D landscape basic platform will be increased or tailored according to the specific application. The general 3D landscape basic platform includes several functional modules such as rendering module, data management module, engine module, and editing module. The system structure shown in Figure 11 is a system structure diagram of several functional modules that should be considered when researching and developing this basic platform. On this platform, the design of $3 \mathrm{D}$ data model, spatial data management, and 3D engine design are the key parts of the platform design. A good platform design should also provide users with flexible editing functions and secondary development interfaces. Users can also customize their own data management mode and choose flexible VR algorithms. 
TABle 1: Evaluation of the design effect of the urban three-dimensional landscape basic platform system.

\begin{tabular}{lcccccccc}
\hline Number & Simulation effect & Design effect & Number & Simulation effect & Design effect & Number & Simulation effect & Design effect \\
\hline 1 & 95.70 & 87.68 & 22 & 94.89 & 73.96 & 43 & 91.02 & 70.58 \\
2 & 95.30 & 89.16 & 23 & 86.14 & 79.14 & 44 & 93.73 \\
3 & 89.84 & 89.09 & 24 & 95.66 & 87.36 & 45 & 95.36 \\
4 & 91.46 & 74.26 & 25 & 93.04 & 88.22 & 46 & 91.71 \\
5 & 96.48 & 76.37 & 26 & 92.08 & 73.53 & 47 & 96.31 & 84.24 \\
6 & 90.90 & 83.84 & 27 & 90.08 & 86.97 & 48 & 92.64 \\
7 & 90.52 & 76.47 & 28 & 93.56 & 83.02 & 49 & 91.06 \\
8 & 89.58 & 69.86 & 29 & 90.55 & 84.11 & 50 & 92.55 \\
9 & 96.54 & 76.26 & 30 & 95.08 & 87.05 & 51 & 90.42 \\
10 & 87.91 & 78.01 & 31 & 94.89 & 77.47 & 52 & 89.17 \\
11 & 86.68 & 76.23 & 32 & 95.62 & 74.27 & 53 & 90.96 \\
12 & 89.82 & 71.63 & 33 & 92.48 & 79.63 & 54 & 95.98 \\
13 & 87.11 & 79.88 & 34 & 94.04 & 89.76 & 55 & 91.59 \\
14 & 87.38 & 90.99 & 35 & 88.75 & 88.22 & 56 & 89.24 \\
15 & 95.95 & 69.93 & 36 & 86.25 & 74.18 & 57 & 86.23 \\
16 & 90.95 & 73.52 & 37 & 92.11 & 71.01 & 58 & 92.02 \\
17 & 90.08 & 87.06 & 38 & 87.24 & 70.74 & 59 & 83.36 \\
18 & 91.49 & 88.16 & 39 & 95.67 & 85.47 & 60 & 98.38 \\
19 & 93.75 & 81.19 & 40 & 95.98 & 76.32 & 61 & 95.18 \\
20 & 88.60 & 88.92 & 41 & 89.76 & 70.55 & 62 & 89.29 \\
21 & 92.28 & 84.98 & 42 & 91.63 & 89.29 & 63 & 96.04 \\
\hline
\end{tabular}

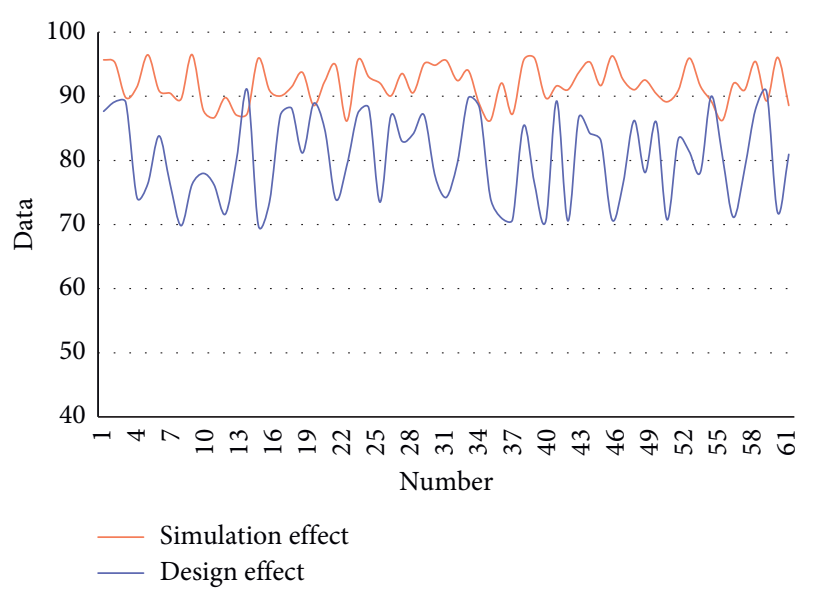

FIgURE 12: Quantitative evaluation statistics.

After constructing the above-mentioned urban landscape three-dimensional structure analysis system based on big data technology and digital technology, the performance of the system is verified. This paper designs multiple groups of urban three-dimensional structures to test the system performance and count the three-dimensional structure simulation effects and actual design effects. The three-dimensional simulation effect is quantitatively analyzed by postdesign evaluation, and the results shown in Table 1 and Figure 12 are obtained.

From the above analysis, the urban landscape threedimensional structure analysis system based on big data technology and digital technology proposed in this paper has good design and simulation effects, and the system designed in this paper can be used for auxiliary research in the threedimensional design and analysis of urban landscape.

\section{Conclusion}

The landscape design business of the Urban Space Planning and Design Institute has been developed so far, and the development environment it faces has also become more complicated. If an enterprise wants to realize its own development, it must have an overall plan for the future development direction, business field, and advantages of the Urban Space Planning and Design Institute. In addition, the Urban Space Planning and Design Institute must consciously adjust its own internal and external environmental changes in strategic planning, so as to better adapt to environmental needs and promote the long-term development of the enterprise. At present, domestic and foreign experts and scholars have insufficient research on the related strategies of urban space planning and design enterprise landscape business development. The research on this topic will help better improve this theory and provide a reference for the development of corporate landscape business in urban space planning in the later period. This paper combines big data technology and digital technology to analyze the threedimensional structure and performance of urban landscapes, which effectively improves the scientific nature of urban landscape design.

\section{Data Availability}

The labeled dataset used to support the findings of this study are available from the corresponding author upon request.

\section{Conflicts of Interest}

The authors declare no conflicts of interest. 


\section{Acknowledgments}

This study was sponsored and supported by the Fundamental Research Funds for the Central Universities: Research on the Visual Evaluation of Winter Landscape in Cold Cities in Northeastern China-with examples of Shenyang and Harbin (PID: 02040022120002).

\section{References}

[1] O. Guerra-Santin and S. Silvester, "Development of Dutch occupancy and heating profiles for building simulation," Building Research \& Information, vol. 45, no. 4, pp. 396-413, 2017.

[2] T. Dodd, C. Yan, and I. Ivanov, "Simulation-based methods for model building and refinement in cryoelectron microscopy," Journal of Chemical Information and Modeling, vol. 60, no. 5, pp. 2470-2483, 2020.

[3] T. Abuimara, W. O’Brien, B. Gunay, and J. S. Carrizo, “Towards occupant-centric simulation-aided building design: a case study," Building Research \& Information, vol. 47, no. 8, pp. 866-882, 2019.

[4] P. Remmen, M. Lauster, M. Mans, M. Fuchs, T. Osterhage, and D. Müller, "TEASER: an open tool for urban energy modelling of building stocks," Journal of Building Performance Simulation, vol. 11, no. 1, pp. 84-98, 2018.

[5] N. Endo, E. Shimoda, K. Goshome, T. Yamane, T. Nozu, and T. Maeda, "Simulation of design and operation of hydrogen energy utilization system for a zero emission building," International Journal of Hydrogen Energy, vol. 44, no. 14, pp. 7118-7124, 2019.

[6] I. Beausoleil-Morrison, "Learning the fundamentals of building performance simulation through an experiential teaching approach," Journal of Building Performance Simulation, vol. 12, no. 3, pp. 308-325, 2019.

[7] C. Xiong, J. Huang, and X. Lu, "Framework for city-scale building seismic resilience simulation and repair scheduling with labor constraints driven by time-history analysis," Computer-Aided Civil and Infrastructure Engineering, vol. 35, no. 4, pp. 322-341, 2020.

[8] A. D. Black, "Wor(l)d-building: simulation and metaphor at the mars desert research station," Journal of Linguistic Anthropology, vol. 28, no. 2, pp. 137-155, 2018.

[9] K. Hanson, L. Hernandez, and J. A. Banaski, "Building simulation exercise capacity in Latin America to manage public health emergencies," Health security, vol. 16, no. S1, pp. S98-102, 2018.

[10] E. K. Wati and N. Widiansyah, "Design of learning media: modeling \& simulation of building thermal comfort optimization system in building physics course," Jurnal Pendidikan IPA Indonesia, vol. 9, no. 2, pp. 257-266, 2020.

[11] C. W. Lee and S.-J. Cho, "The development of converting program from sealed geological model to Gmsh, COMSOL for building simulation grid," Journal of the Korean Earth Science Society, vol. 38, no. 1, pp. 80-90, 2017.

[12] C. Miller, D. Thomas, J. Kämpf, and A. Schlueter, "Urban and building multiscale co-simulation: case study implementations on two university campuses," Journal of Building Performance Simulation, vol. 11, no. 3, pp. 309-321, 2018.

[13] X. Xie and Z. Gou, "Building performance simulation as an early intervention or late verification in architectural design: same performance outcome but different design solutions," Journal of Green Building, vol. 12, no. 1, pp. 45-61, 2017.
[14] A. I. Adilkhodjayev, I. M. Mahamataliev, and S. S. Shaumarov, "Theoretical aspects of structural and simulation modeling of the macrostructure of composite building materials," Journal of Tashkent Institute of Railway Engineers, vol. 14, no. 2, pp. 3-14, 2019.

[15] S. Imam, D. A. Coley, and I. Walker, "The building performance gap: are modellers literate?" Building Service Engineering Research and Technology, vol. 38, no. 3, pp. 351-375, 2017.

[16] J.-S. Pei, B. Carboni, and W. Lacarbonara, "Mem-models as building blocks for simulation and identification of hysteretic systems," Nonlinear Dynamics, vol. 100, no. 2, pp. 973-998, 2020.

[17] A. Brunelli, F. de Silva, A. Piro et al., "Numerical simulation of the seismic response and soil-structure interaction for a monitored masonry school building damaged by the 2016 Central Italy earthquake," Bulletin of Earthquake Engineering, vol. 19, no. 2, pp. 1181-1211, 2021.

[18] P. Andrio, A. Hospital, J. Conejero et al., "Bioexcel building blocks, a software library for interoperable biomolecular simulation workflows," Scientific Data, vol. 6, no. 1, pp. 169-184, 2019.

[19] G. Petrou, A. Mavrogianni, P. Symonds et al., "Can the choice of building performance simulation tool significantly alter the level of predicted indoor overheating risk in London flats?" Building Service Engineering Research and Technology, vol. 40, no. 1, pp. 30-46, 2019.

[20] S. Gibeaux, C. Thomachot-Schneider, S. Eyssautier-Chuine, B. Mari, and P. Vazquez, "Simulation of acid weathering on natural and artificial building stones according to the current atmospheric $\mathrm{SO}_{2} / \mathrm{NO}_{x}$ rate," Environmental Earth Sciences, vol. 77, no. 9, pp. 1-19, 2018. 